\title{
Expressing the Behavior of Three Very Different Concurrent Systems by Using Natural Extensions of Separation Logic
}

\author{
Edgar G. Daylight \\ a.k.a. K. Van Oudheusden, \\ Institute of Logic, Language, and Computation, \\ University of Amsterdam, The Netherlands \\ egdaylight@yahoo.com
}

\author{
Sandeep K. Shukla \\ Department of Electrical \\ \& Computer Engineering, \\ Virginia Tech., USA \\ shukla@vt.edu
}

\author{
Davide Sergio \\ Institute of Logic, Language, and Computation, \\ University of Amsterdam, The Netherlands \\ D.Sergio@student.uva.nl
}

\begin{abstract}
Separation Logic is a non-classical logic used to verify pointer-intensive code. In this paper, however, we show that Separation Logic, along with its natural extensions, can also be used as a specification language for concurrent-system design. To do so, we express the behavior of three very different concurrent systems: a Subway, a Stopwatch, and a $2 \times 2$ Switch. The Subway is originally implemented in LUSTRE, the Stopwatch in Esterel, and the $2 \times 2$ Switch in Bluespec.
\end{abstract}

\section{Introduction}

Concurrent systems, specified today, can have very different properties. Depending on these properties, a practical specification language is chosen. For instance, consider a designer who can choose between the synchronous language Esterel and the guarded-command language Bluespec in order to specify the modal behavior of a Stopwatch, on the one hand, and the shared-memory behavior of a $2 \times 2$ Switch, on the other hand. The designer will typically choose Esterel for the Stopwatch and Bluespec for the $2 \times 2$ Switch and not the other way around. In other words, while it is of course theoretically possible to express modal behavior with Bluespec and shared-memory behavior with Esterel, it is -in terms of practical expressiveness- not interesting to do so.

The statements in the previous paragraph are based on "common design experience", not on a formal metric of practical expressiveness. To the best of our knowledge, such a metric is not available in the current literature 1 . In this paper we do not try to find such a metric either, for we believe it is wiser to first obtain many specifications of various systems using different specification languages and to compare them based on intuitive notions of "practical expressiveness". Based on these informal comparisons, we can then search for a metric that is both well defined and practically relevant.

In this paper we choose the formalism of Separation Logic and its natural extensions to express the behavior of three very different systems:

- A Subway system, originally specified with LUSTRE [8].

- A Stopwatch, originally specified with Esterel [7].

- A $2 \times 2$ Switch, originally specified with Bluespec [2].

\footnotetext{
${ }^{1}$ Note that the conciseness of a specification is too simplistic a metric: a lengthy specification $S_{0}$ can be preferred over a short specification $S_{1}$ if, for instance, $S_{0}$ explicitly captures a design requirement that is only implicitly present in $S_{1}$.

S. Fröschle, D. Gorla (Eds.): Workshop on Expressiveness in Concurrency 2009 (EXPRESS'09).

EPTCS 8, 2009, pp. 26-40 doi 10.4204/EPTCS.8.3

(c) E.G. Daylight \& S.K. Shukla \& D. Sergio

This work is licensed under the Creative Commons Attribution License.
} 


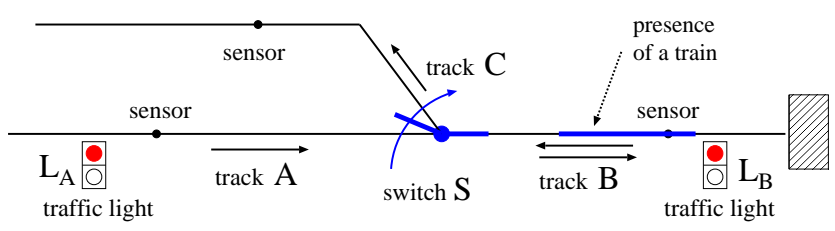

Figure 1: A scenario of the Subway system

Our specifications are based on an analogy that we make with photography, explained below. The analogy is formalized by means of Separation Logic [13, 15]. This logic, in turn, is an extension of Hoare Logic and is typically not used in the way we use it in this paper, i.e. as a specification language.

\section{An Analogy with Photography}

Given a concurrent system such as the Subway system in Figure 1, we make the following analogy with photography. Let various photographers be assigned to different locations in Figure 1, By taking consecutive camera snapshots, each photographer captures local change of some part of the Subway. Then, by combining all local changes, we obtain a complete specification of the Subway.

For instance, suppose photographer $\mathrm{Ph} 1$ is assigned to take snapshots of track A in Figure 1 while photographer $\mathrm{Ph} 2$ is assigned to track $\mathrm{B}$. Ph1 can, by taking one snapshot, either observe the presence of a train on track $\mathrm{A}$, denoted by $1 @ A$, or the vacancy of track $\mathrm{A}$, denoted by $0 @ A$. By taking two consecutive snapshots, Ph1 can observe four possible changes: ( $a @ A, b @ A)$ with $a, b \in\{0,1\}$-where we shall use $(a, b) @ A$ to abbreviate $(a @ A, b @ A)$. For example, Ph1 may observe the arrival of a train on A, denoted by $(0,1) @ A$. Likewise, Ph2 may observe the continuous vacancy of track B, denoted by $(0,0) @ B$. By combining the observations of Ph1 and Ph2, we obtain the composite change $(0,1) @ A *$ $(0,0) @ B$, describing a system in which a train arrives on A while, simultaneously, track B is vacant.

The example, presented above, can be extended by adding more photographers, as we shall illustrate in Section 2 when discussing the Subway in more detail. In addition, we can generalize the notions of 'snapshot' and 'change' to the notion of 'change of change'. This extension will be needed when specifying the modal behavior of a Stopwatch in Section 3. In terms of the analogy, the photographer capturing a scene by means of 'change', has become a camera man, capturing the change from one scene to another. Another generalization is needed when specifying the $2 \times 2$ Switch in Section 4 There, the concept of 'snapshot' is generalized to that of an 'hierarchical snapshot', implying that each photographer can zoom in on specific details of the concurrent system under investigation. Consequently, hierarchical change is used (instead of plain change) to capture the concurrent behavior of the Switch.

\section{Related Work}

Our analogy with photography is formalized in this paper by using the following embarrassingly simple logics. First, the Logic of Snapshots is merely an instance of Separation Logic's assertion language using the @ primitive of Ahmed et al. [1] instead of the usual points-to predicate [13, 15]. The key point is that formulae denote unary predicates over snapshots (shot) of the system state. The second logic is ChaLo, the Logic of Change. It is basically Yang's "Relational Separation Logic" [16] where formulae denote binary relations $c h a$ of the form $\left(\right.$ shot $_{\text {in }}$, shot $\left._{\text {out }}\right)$ rather than unary predicates. For notational convenience

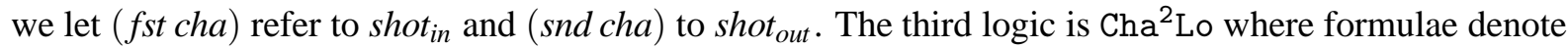
relations on relations over snapshots, i.e. sets of elements of the form: $\left(c h a_{i n}, c h a_{\text {out }}\right)$. But the semantics 
will require that $\left(s n d c h a_{\text {in }}\right)$ and $\left(f s t c h a_{\text {out }}\right)$ are always equal (or else completely irrelevant), so formulae actually denote triples of snapshots. Hence, $\mathrm{Cha}^{2} \mathrm{Lo}$ is a straightforward adaptation of ChaLo from binary to ternary relations. Continuing in the same manner, we then present $\mathrm{Cha}^{4} \mathrm{Lo}$, denoting elements of the form: $\left(\left(c h a_{1}, c h a_{2}\right),\left(c h a_{3}, c h a_{4}\right)\right)$ where $\left(s n d c h a_{2}\right)$ is equal to $\left(f s t c h a_{3}\right)$. Further extrapolation results in Cha ${ }^{8} \mathrm{Lo}$ and, in general, in Cha ${ }^{\mathrm{n}} \mathrm{Lo}$ with $\mathrm{n}=2^{k}$ and $k$ a strictly positive integer. In summary, it is more the use of the logical definitions that is new and interesting, rather than the definitions themselves.

The formalism in this paper abides by the Synchronous Hypothesis [3, 14]. To illustrate this, consider $(0,1) @ A *(G, R) @ L_{A}$, which can be read operationally as follows: "When track A's sensor senses the arrival of a train, the Subway system responds by turning traffic light $\mathrm{L}_{\mathrm{A}}$ from green $(G)$ to red $(R)$." Operationally, it makes no sense to reason in the opposite direction; i.e. by starting with the light and concluding with A's sensor. Thus, we have an ordering from $(0,1) @ A$ to $(G, R) @ L_{A}$. But, since $*$ requires that both occur simultaneously, $(G, R) @ L_{A}$ has to be an instantaneous reaction to $(0,1) @ A$.

The analogy with photography, resulting in the concepts of 'snapshot', 'change', and 'change of change', sets it apart from other well-established formalisms, such as Statecharts [9], Communicating Sequential Processes [10], the $\pi$-calculus [12], spatial logics (e.g. [4]), and process algebras [6], just to name a few. Lack of space prevents us from delving into these other formalisms here.

\section{Subway}

We introduce the Logic of Snapshots and its extension ChaLo (i.e. the Logic of Change) to specify a Subway. The Logic of Snapshots is system dependent. That is, we shall introduce syntax for snapshots that depends on the Subway. Later, when discussing the Stopwatch (Section 3) and the Switch (Section 4), we shall introduce other syntax. ChaLo, on the other hand, is only defined once.

This section consists of three parts. Section 2.1 presents the design intent of the Subway. Section 2.2 illustrates how the Logic of Snapshots and ChaLo can be used to specify the Subway. Finally, Section 2.3 presents the formalization.

\subsection{Design Intent}

The objective in Figure 1 is to design a Subway system so that a train can enter by track A, temporarily use track B, and then leave by track C [8]. At all times, at most one train is present in the Subway system. Seven state elements constitute the system. Four state elements are inputs: the sensor values of the tracks A, B, and C, and the switch S. Three state elements are outputs: the actuator of the switch S and the two traffic lights $\mathrm{L}_{A}$ and $\mathrm{L}_{B}$. Each state element is presented below along with its possible values: sensors of $\mathrm{A}, \mathrm{B}$, and $\mathrm{C}$

$0 \quad 1$

(iii) $\quad L_{A}, L_{B}$

$A B \quad$ off $\quad B C$

When a train is on a track (e.g. track B in Figure 1), then the corresponding sensor value is 1, else it is 0 . The sensor of switch $\mathrm{S}$ has the value $A B$ when tracks $\mathrm{A}$ and $\mathrm{B}$ are connected and $B C$ when tracks $\mathrm{B}$ and $\mathrm{C}$ are connected. The value off occurs when no tracks are connected, as is the case in Figure 1 The actuator of switch $\mathrm{S}$ has the value $A B$ when the switch is being steered in order to (eventually) connect tracks A and B. Similarly, the value is $B C$ when connecting tracks B and C, as illustrated in Figure 1 by the arrowed arc. The actuator has the value off when the switch is not being steered (i.e. typically when two tracks are connected). Traffic lights can either be green $(G)$ or red $(R)$. Green light $\mathrm{L}_{\mathrm{A}}$ allows a train to enter track $A$ from the left. Green light $L_{B}$ allows a train to depart from track B by moving backwards (preferably onto track $\mathrm{C}$ !). 


\subsection{Some Specifications}

We now illustrate the Logic of Snapshots and ChaLo by presenting some specifications of the Subway. Since the complete formalization of the Logic of Snapshots is straightforward but lengthy, we merely illustrate it below. The more important logic ChaLo, on the other hand, is illustrated below and formally defined in Section 2.3

As a first example, consider the following snapshot specification in the Logic of Snapshots:

$$
0 @ A * 1 @ B * G @ L_{B} * B C @ S e n S
$$

It partially describes a particular instance of the Subway: track A is vacant, track B is occupied, traffic light $\mathrm{L}_{\mathrm{B}}$ is green $(G)$ and hence granting exit to the train on track $\mathrm{B}$. The switch $\mathrm{S}$ is, based on its sensor $(\operatorname{Sen} S)$, connecting track B with track $\mathrm{C}$. The snapshot is partial because it does not capture the status of track $\mathrm{C}$, the traffic light $\mathrm{L}_{\mathrm{A}}$, and the actuator of the switch $\mathrm{S}$.

Expression (1) is a syntactic abbreviation for:

(2) $\quad<0 @ A * 1 @ B, B C @ S e n S, e m p, G @ L_{B}>$

which is a tuple of four snapshot expressions. The first entry $0 @ A * 1 @ B$ describes the states of the tracks, the second describes the switch's sensor, the third describes the switch's actuator, where emp abbreviates "empty", and the fourth describes the traffic lights. The meaning of (2) is described next.

Let $\operatorname{Tr}=\{$ false, true $\}$ denote the set of truth values, Var a set of variables, and Val a set of values: $V a l=\{0,1\} \cup\{A B, B C$,off $\} \cup\{R, G\}$ and $V a l_{\perp}=\operatorname{Val} \cup\{\perp\}$. The set of assignment functions is Asgmt $:=\operatorname{Var} \rightarrow \operatorname{Val}_{\perp}$. Let $s$ denote an assignment function, i.e. $s \in A s g m t$. Then, the semantical interpretation of (2), using $s$, results in a semantic snapshot $\left(\right.$ shot $_{1}$, shot $_{2}$, shot $_{3}$, shot $\left._{4}\right)$ :

$$
\begin{aligned}
& s,\left(\text { shot }_{1}, \text { shot }_{2}, \text { shot }_{3}, \text { shot }_{4}\right) \\
& =<0 @ A * 1 @ B, B C @ S e n S, e m p, G @ L_{B}> \\
& \text { iff } \quad s, \text { shot }_{1}=0 @ A * 1 @ B \text { and } \\
& s, \text { shot }_{2}=B C @ \operatorname{SenS} \quad \text { and } \\
& s, \text { shot }_{3}=e m p \quad \text { and } \\
& s, \text { shot }_{4} \models G @ L_{B}
\end{aligned}
$$

That is, each local semantic snapshot shot $_{i}$ with $i \in\{1,2,3,4\}$ models the corresponding syntactic snapshot as a function. I.e., shot $_{1}$ is a function that maps $A$ to $0, B$ to 1 , and $C$ to $\perp$. Function $s t_{2}$ maps $S e n S$ to $B C$. Function shot $_{3}$ maps $A c t S$ to $\perp$, since no information ("empty" emp) is present about the actuator $A c t S$. Function $s t_{4}$ maps $L_{A}$ to $\perp$ and $L_{B}$ to $G$. Finally, we remark that $\left(\right.$ shot $_{1}$, shot $_{2}$, shot $_{3}$, shot $\left._{4}\right) \in$ Snshot where Snshot is the domain of semantic snapshots.

As a second example, we illustrate the difference between Separation Logic's spatial conjunction $*$ and classical conjunction $\wedge$. Let us take (5) and replace $*$ by $\wedge$, then we have:

$$
\begin{aligned}
& s, \text { shot }_{1}=0 @ A \wedge 1 @ B \\
& \text { iff } \quad s, \text { shot }_{1}=0 @ A \text { and } \\
& s, \text { shot }_{1}=1 @ B
\end{aligned}
$$

Now, (10) states that shot $_{1}$ is a function mapping $A$ to 0 and $B$ and $C$ to $\perp$. On the other hand, (11) states that shot $t_{1}$ maps $B$ to 1 and $A$ and $C$ to $\perp$. This is clearly not possible, so $\wedge$ is used incorrectly in (9).

The previous example shows that $\wedge$ can not replace $*$ without altering the intended meaning. This is due to the chosen semantics of @: 0@A only describes the state of track A. In the alternative classical semantics, 0@A would describe the complete state of all three tracks in the Subway system, with the additional knowledge that track $A$ is vacant 2 . The same arguments also hold for change, such as $(0,1) @ A$.

\footnotetext{
${ }^{2}$ It is of course possible to avoid the use of $*$ in this paper by redefining the meaning of @ in accordance to the classical semantics, but the purpose of this paper is to use Separation Logic for case studies, such as the Subway system, for which it was not initially intended.
} 
For a third example, recall the photographers $\mathrm{Ph} 1$ and $\mathrm{Ph} 2$ from Section 1 . When both photographers combine their observations, they conclude that, in accordance to a correctly-behaving Subway system, the following implication has to hold: $(0,1) @ A \rightarrow(0,0) @ B$. In words: if a train arrives on track $A$, then, at the same time, track B remains vacant. That is, it is impossible for Ph1 to observe $(0,1) @ A$ while Ph2 observes, say, $(1,0) @ B$.

The implication $(0,1) @ A \rightarrow(0,0) @ B$ is an abbreviation for: $(0,1) @ A * \exists x \exists y(x, y) @ B \Rightarrow \exists x^{\prime} \exists y^{\prime}\left(x^{\prime}, y^{\prime}\right) @ A *(0,0) @ B$, where we have ensured that the same state elements $(A$ and $B)$ are present on the left- and righthand side of $\Rightarrow$. That is, $\rightarrow$ is defined here (by example) in terms of $\Rightarrow$, which, in turn, is defined formally in the next section (cf. Table1).

As a fourth and final example, consider $(0,1) @ A \leftrightarrow(G, R) @ L_{A}$, which is an abbreviation for $\left[(0,1) @ A \rightarrow(G, R) @ L_{A}\right] \wedge\left[(G, R) @ L_{A} \rightarrow(0,1) @ A\right]$. It describes the arrival of a train on A while traffic light $\mathrm{L}_{\mathrm{A}}$ turns from green to red. Using this, $\mathrm{Ph} 1, \mathrm{Ph} 2$, and the photographer of light $\mathrm{L}_{\mathrm{A}}$ can combine $(\otimes)$ their observations as follows:

$$
[(0,1) @ A \rightarrow(0,0) @ B] \otimes\left[(0,1) @ A \leftrightarrow(G, R) @ L_{A}\right]
$$

From this we can, for instance, deduce that $(G, R) @ L_{A}$ implies $(0,0) @ B$.

Similar to $*$ and $\rightarrow$, the use of $\otimes$ aids us in obtaining a short formal exposition. It could be completely avoided by only using $*$ and $\wedge$ but at the cost of longer specifications. It too is formally defined in Table 1

\section{Additional Notation}

Let $X_{\perp}:=X \cup\{\perp\}$. For $f:: A_{\perp} \rightarrow B_{\perp}$ we write $f=\underline{\lambda} x . \alpha$ to denote the mapping: $f(\perp)=\perp$ and $f(a)=[a / x] \alpha$ for $a \in A$. Also, the domain $(\operatorname{dom} f)$ of a partial function $f$ is the set of $x$ 's such that $f(x)$ does not equal $\perp$. In particular, $(\operatorname{dom}(\lambda x . \perp))=\emptyset$. Finally, for domains $D$ and $E$, let $[D \rightarrow E]$ denote the set $\{f \mid f:: D \rightarrow E\}$. Consider functions $f, g \in\left[D \rightarrow E_{\perp}\right]$. We define the operations $\sharp$ and . as follows:

$\sharp \quad:: \quad\left(D \rightarrow E_{\perp}\right)_{\perp} \rightarrow\left(D \rightarrow E_{\perp}\right)_{\perp} \rightarrow \operatorname{Tr}_{\perp}$

$\sharp:=\underline{\lambda} f \cdot \underline{\lambda} g .(\operatorname{dom} f) \cap(\operatorname{dom} g)==\emptyset$

- $:: \quad\left(D \rightarrow E_{\perp}\right)_{\perp} \rightarrow\left(D \rightarrow E_{\perp}\right)_{\perp} \rightarrow\left(D \rightarrow E_{\perp}\right)_{\perp}$

- $:=\underline{\lambda} f . \underline{\lambda} g$. if $f \sharp g$ then $f \cup g$ else $\perp$

For example, if we revisit the partial function $s h o t_{1}$ in (5). Then $s h o t_{1}=s h o t_{1}^{a}$. shot ${ }_{1}^{b}$ where $s h o t_{1}^{a}$ is a function that maps $A$ to 0 and $B$ and $C$ to $\perp$. Likewise, shot $t_{1}^{b}$ maps $B$ to 1 and $A$ and $C$ to $\perp$. Clearly, shot $_{1}^{a}$ and shot ${ }_{1}^{b}$ have disjoint domains: shot $t_{1}^{a} \sharp$ shot $_{1}^{b}$.

\subsection{Logic of Change}

We are now in a position to present ChaLo, the Logic of Change. After taking the following four remarks into account, Table 1 can be consulted.

First, we define semantical change as a pair of semantical snapshots:

ch $\in$ Change $:=$ Snshot $\times$ Snshot

Second, given semantical changes $c h_{1}$ and $c h_{2}$, the disjointness $(\sharp)$ and the combination (.) of $c h_{1}$ and $c h_{2}$ can be defined:

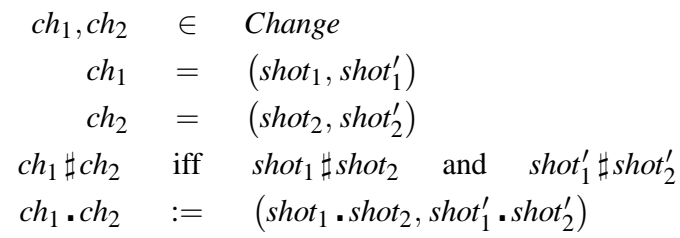


Table 1: ChaLo

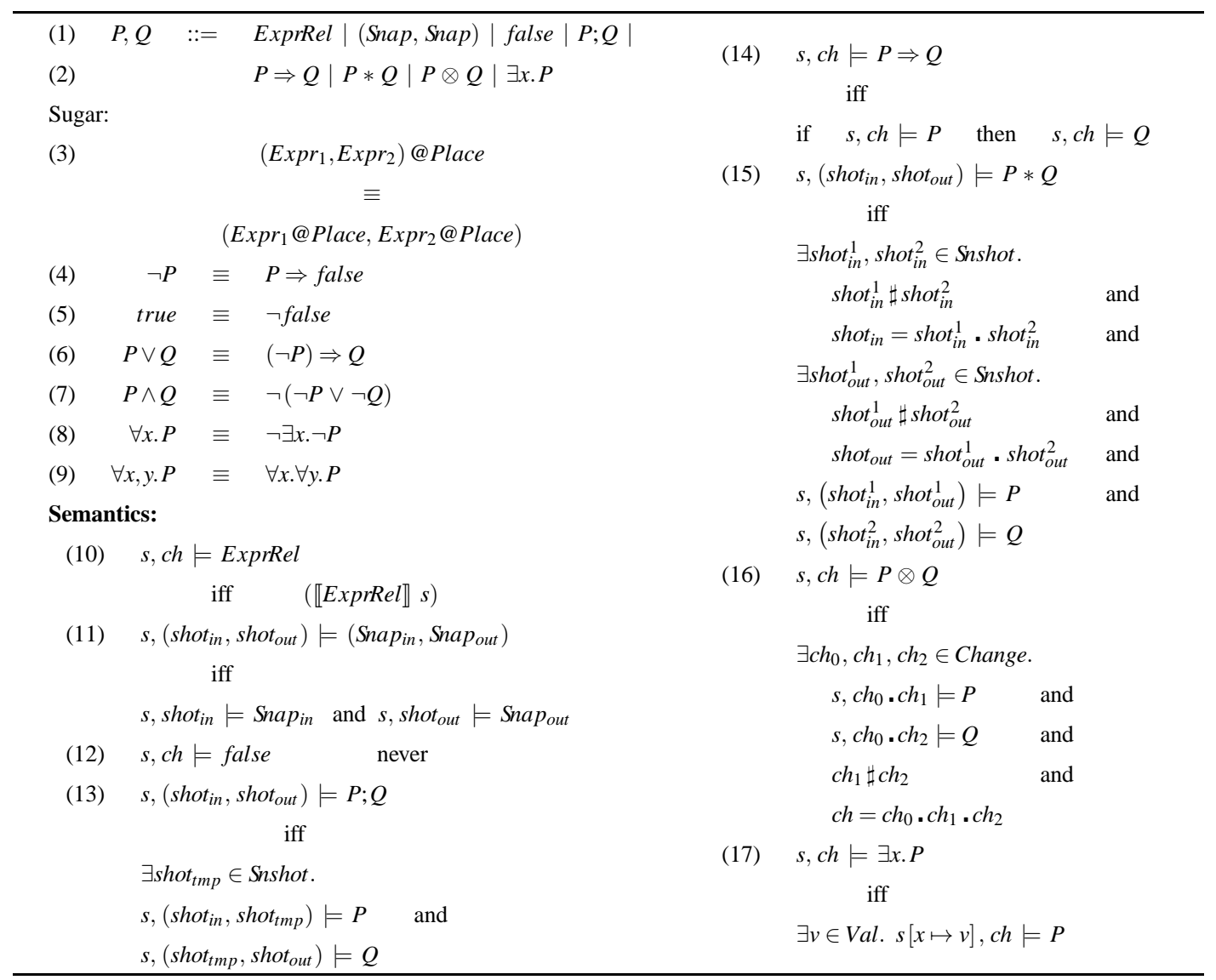

Third, the semantics of a ChaLo formula $P$ is of the form:

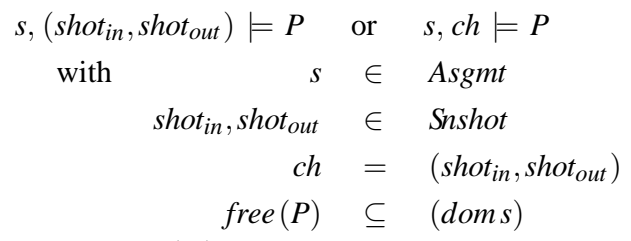

where free $(P)$ denotes the free variables in $P$. Fourth, an example of an expression relation ExprRel is $x=1$ and its valuation $(\llbracket$ ExprRel $\rrbracket s)$ amounts to checking whether $(s x)=1$ holds. The trivial definition of $\llbracket$ ExprRel $\rrbracket$ is omitted from this paper.

\section{Stopwatch}

Our second case study is a Stopwatch, introduced in Section 3.1. To capture its behavior, we shall introduce the Logic of Change of Change $\left(\mathrm{Cha}^{2} \mathrm{Lo}\right)$ and similar extensions (Cha $\left.{ }^{4} \mathrm{Lo}, \mathrm{Cha}{ }^{8} \mathrm{Lo}, \ldots\right)$ in Section 3.2. Finally, various specifications of the Stopwatch's behavior are presented in Section 3.3 


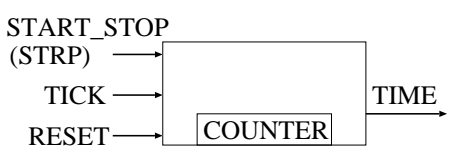

(i)

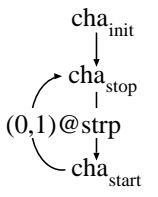

(ii)

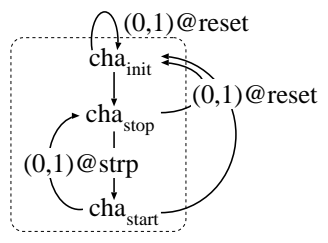

(iii)

Figure 2: (i) The Stopwatch, (ii) the $\mathrm{Cha}^{2} \mathrm{Lo}$ diagram for $c h a_{b a s i c}^{2}$, and (iii) the Cha ${ }^{4} \mathrm{Lo}$ diagram for cha $a_{\text {reset }}$.

\subsection{Design Intent}

The design intent of the Stopwatch is too lengthy to present in plain English. Therefore, we let our specifications speak for themselves. They can also be checked against the original Esterel specification, presented in [7].

The Stopwatch in Figure 2(i) can be briefly described as follows. The input signals START_STOP (or STRP for short), TICK, and RESET are immutable elements. That is, their value is completely determined by the external behavior of the Stopwatch. In fact, STRP and RESET are buttons which are pressed (1) or depressed ( 0 ) by the user, and TICK is the signal ( 0 or 1$)$ of an external clock. The internal register COUNTER and the output signal TIME are mutable elements. That is, their value is determined by the internal behavior of Stopwatch. Finally, we also use an internal register MODE, not shown in Figure 2 to book-keep the current mode of execution. It too is a mutable element.

The locations, presented above, can be assigned to the photographers. We present six examples. First, $(0,1) @ s t r p$, describing change from 0@strp to $1 @ s t r p$, captures the behavior of a user who presses the STRP button. Second, $(0,1) @ \operatorname{strp} *(0,1) @$ reset describes a user who simultaneously presses both the STRP and RESET buttons. Third, $(x, x+1) @$ time expresses an increase of TIME from $x$ to $x+1$. Fourth, $(x, a b s) @$ time expresses the sending of $x$ to TIME, followed by not sending anything to TIME (i.e. an "absent" signal). In general, $(a, b) @$ time is syntactically correct when $a, b \in \mathbb{N} \cup\{a b s\}$. Fifth, $(0,1) @$ tick describes a positive TICK. In general, $(a, b) @$ tick is syntactically correct when $a, b \in\{0,1\}$. Sixth, (init,stop)@mode expresses that the system changes from mode init to mode stop. In general, $(a, b) @$ mode is correct when $a, b \in\{$ init, stop, start $\}$; its intended meaning will become clear later.

\subsection{Logic of Change of Change and Beyond}

The Stopwatch is a prime example of modal behaviour: pressing a button of the Stopwatch can have a different effect, depending on the mode of operation. While the other two case studies in this paper only contain one mode of operation, the Stopwatch contains several: $c h a_{i n i t}, c_{\text {stop }}, \operatorname{cha}_{\text {start }}, \operatorname{cha}_{\text {basic }}^{2}$, cha $_{\text {reset }}^{4}$, ... We present some intuition about these modes, before defining the Logic of Change of Change (i.e. $\mathrm{Cha}^{2} \mathrm{Lo}$ ) and its extensions. The meaning of each mode will become apparent in Section 3.3 .

The modes $c h a_{\text {init }}$, cha $a_{\text {stop }}$, and $c h a_{\text {start }}$ are expressed as simple ChaLo formulae. Mode $c h a_{b a s i c}^{2}$, on the other hand, is expressed in Cha ${ }^{2} \mathrm{Lo}$, describing transformations between modes $c h a_{i n i t}, c h a_{\text {stop }}$, and cha $a_{\text {start }}$. That is, $c h a_{\text {basic }}^{2}$ describes an hierarchical mode, containing the simpler modes $c h a_{\text {init }}, c h a_{\text {stop }}$, and $c h a_{\text {start }}$, as is illustrated graphically in Figure 2(ii). Formula $c h a_{\text {reset }}^{4}$ is expressed in Cha ${ }^{4}$ Lo and describes transformations between $\mathrm{Cha}^{2} \mathrm{Lo}$ formulae. That is, $c h a_{\text {reset }}^{4}$ describes an hierarchical mode, containing simpler modes (e.g. $c h a_{\text {basic }}^{2}$ ), as is graphically illustrated in Figure 2(iii). This hierarchi- 
Table 2: Cha ${ }^{2}$ Lo

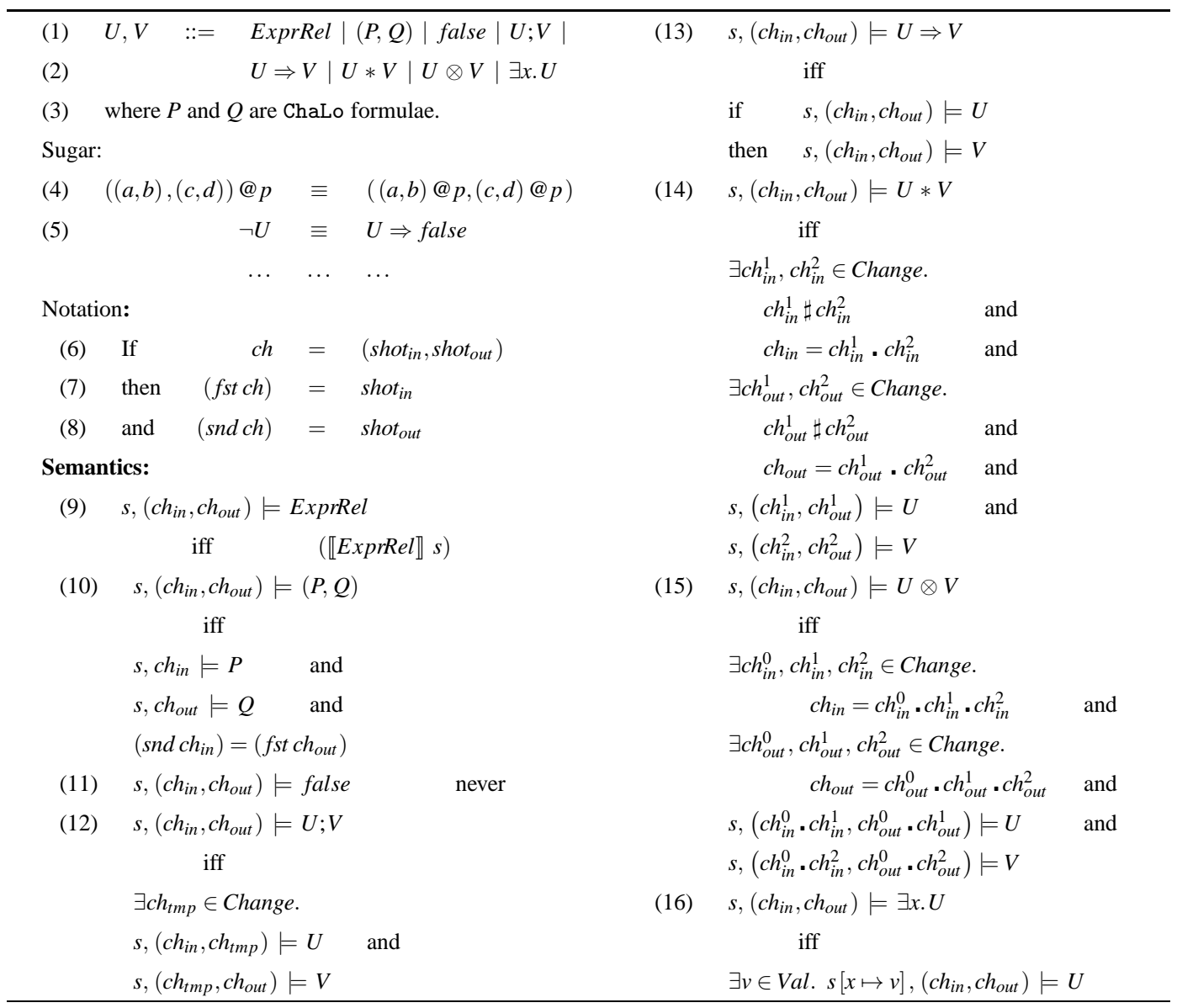

cal extrapolation continues with $\mathrm{Cha}^{8} \mathrm{Lo}$ formula $c h a_{\text {lap }}^{8}$, describing transformations between Cha ${ }^{4} \mathrm{Lo}$ formulae. In general, we deal with a Cha ${ }^{\mathrm{n}} \mathrm{Lo}$ formula with $\mathrm{n}=2^{k}$ where $k$ is strictly positive.

A Cha ${ }^{2} \mathrm{Lo}$ formula $U$ is semantically interpreted as a pair of changes:

$s,\left(c_{\text {in }}, c h_{\text {out }}\right) \models U$ with: $s \in A s g m t$ and $\operatorname{free}(U) \subseteq($ doms $)$ and

$$
\text { ch }_{\text {in }}, \text { ch }_{\text {out }} \in \text { Change }:=\text { Snshot } \times \text { Snshot }
$$

The pair $\left(c h_{\text {in }}, c h_{\text {out }}\right)$, called a transformation, denotes the change of $c h_{\text {in }}$ into $c h_{\text {out }}$. The definition of $\mathrm{Cha}^{2}$ Lo in Table 2 is self explanatory; we stress the similarity with ChaLo in Table 1

Continuing in the same manner, a Cha ${ }^{4} \mathrm{Lo}$ formula, such as $(U, V)$, is semantically interpreted as a pair of a pair of changes:

$$
\begin{aligned}
& s,\left(\left(c h_{1}, c h_{2}\right),\left(c h_{3}, c h_{4}\right)\right) \models(U, V) \\
& \quad \text { iff } \\
& s,\left(c h_{1}, c h_{2}\right) \models U \quad \text { and } \quad s,\left(c h_{3}, c h_{4}\right) \models V \quad \text { and } \quad\left(s n d c h_{2}\right)=\left(f s t c h_{3}\right)
\end{aligned}
$$

where $U$ and $V$ are $C h a^{2}$ Lo formulae. Cha ${ }^{4}$ Lo's complete definition is obvious and omitted from this paper. The same remark holds for Cha ${ }^{8} \mathrm{Lo}$ (a pair of a pair of a pair of changes) or, in general, Cha ${ }^{\mathrm{n}} \mathrm{Lo}$ with $\mathrm{n}=2^{k}$ where $k$ is strictly positive. The logics Cha ${ }^{2} \mathrm{Lo}$ and Cha ${ }^{4} \mathrm{Lo}$ are used in Section 3.3 to capture the preemption mechanisms of the Stopwatch. 


\section{Conventions}

We present two conventions. First, an underscore denotes a don't care value. E.g., (_,0)@ counter abbreviates $\exists x(x, 0) @$ counter. Likewise, (_, _) @ counter abbreviates $\exists x \exists y(x, y) @$ counter.

Second, similar to Section 2.2 the implication $(0,1) @ \operatorname{strp} \rightarrow(0,0) @$ reset abbreviates: $(0,1) @ \operatorname{strp} *(-,-) @$ reset $\Rightarrow(-,), @ \operatorname{str} p *(0,0) @$ reset.

The previous remark holds for any of the logics. Consider for instance $\mathrm{Cha}^{2} \mathrm{Lo}$ and the following expression:

$\left((0,1),\left({ }_{-},\right)\right) @ s t r p \rightarrow\left(c h a_{\text {stop }}\right.$, cha $\left.a_{\text {start }}\right) \vee\left(c h a_{\text {start }}\right.$, cha $\left.a_{\text {stop }}\right)$ and suppose $c h a_{\text {stop }}$ and $c h a_{\text {start }}$ only describe changes of TICK, COUNTER, and TIME. Then this expression is an abbreviation for:

$\left((0,1),\left({ }_{-},-\right)\right) @ \operatorname{strp} *\left(\left({ }_{-},-\right),(-,-)\right) @$ tick*

$((-,-),(-,-)) @$ counter $*((-,-),(-,-)) @$ time

$\Rightarrow((-,-),(-,-)) @ \operatorname{strp} *\left[\left(c h a_{\text {stop }}\right.\right.$, cha $\left.a_{\text {start }}\right) \vee\left(\right.$ cha $a_{\text {start }}$, cha $\left.\left.a_{\text {stop }}\right)\right]$

\subsection{Some Specifications}

We start by specifying the behavior of a Basic Stopwatch, which is similar to the Stopwatch in Figure2(i) except that the RESET button is excluded. The Basic Stopwatch's behavior is visualized by the Cha ${ }^{2}$ Lo diagram in Figure 2(ii). The diagram distinguishes between three modes of operation: $c h a_{\text {init }}$, cha $a_{\text {stop }}$, and $c h a_{\text {start }}$. After an initialization phase, corresponding to $c h a_{\text {init }}$, the system enters a loop, executing either mode $c h a_{\text {stop }}$ or $c h a_{\text {start }}$, depending on the user's input. That is, by pressing STRP, the Basic Stopwatch transitions from mode $c h a_{\text {stop }}$ to $c h a_{\text {start }}$ or vice versa. This is expressed in Figure 2 (ii) by the label $(0,1) @$ strp. On the other hand, if STRP is not pressed, the Basic Stopwatch stays in its current mode (i.e. $c h a_{\text {stop }}$ or $c h a_{\text {start }}$ ).

The three modes are clarified as follows. First, mode $c h a_{\text {init }}$ amounts to setting COUNTER to the value 0 . That is:

cha $a_{\text {init }}:=($ init,.$) @$ mode $*(-, 0) @$ counter

Note also that mode book-keeps the current mode, which in this case is init. Second,

(1) $\quad$ cha $a_{\text {stop }}:=$ cha $a_{\text {stop }}^{\text {emit }} ;$ cha $a_{\text {stop }}^{\text {await }}$.

The first change $c h a_{\text {stop }}^{\text {emit }}$ expresses that the value of the COUNTER stays the same and it's value $x$ has to be emitted to TIME:

cha $a_{\text {stop }}:=($ stop,,$) @$ mode $* \exists x .[(x, x) @$ counter $*(-, x) @$ time $]$

Since the value $x$ only has to be emitted once, $c h a_{\text {stop }}^{\text {emit }}$ is immediately followed in (1) by $c h a_{\text {stop }}^{\text {await }}$, which expresses that an absent signal $a b s$ is sent to TIME:

ch $a_{\text {stop }}^{\text {await }}:=\left(\right.$ stop,$\left._{-}\right) @$ mode $* \exists x .\left[(x, x) @\right.$ counter $*\left({ }_{-}\right.$, abs $) @$ time $]$

Third,

(2) $\quad$ cha $a_{\text {start }}:=$ cha $a_{\text {start }}^{1} \wedge$ cha $a_{\text {start }}^{2}$.

The first conjunct $c h a_{\text {start }}^{1}$ expresses that, at every positive TICK, the value of COUNTER is incremented by one (from $x-1$ to $x$ ) and sent to TIME:

$$
\begin{aligned}
\text { cha } a_{\text {start }}^{1}:= & (\text { start },-) @ \text { mode } *(0,1) @ \text { tick } * \\
& \exists x .[(x-1, x) @ \text { counter } *(-, x) @ \text { time }]
\end{aligned}
$$

The second conjunct in (2) states that, in the absence of a positive TICK, the value of COUNTER remains constant and an absent signal is sent as output:

$$
\begin{aligned}
c h a_{\text {start }}^{2}:= & (\text { start },-) @ \text { mode } *[(0,0) @ \text { tick } \vee(1, \ldots) @ \text { tick }] * \\
& \exists x .(x, x) @ \text { counter } *(-, \text { abs }) @ \text { time }
\end{aligned}
$$


Having defined the ChaLo formulae, we now define the $\mathrm{Cha}^{2} \mathrm{Lo}$ formulae of Figure 2 (ii) in three steps. First, transformation transf $_{1}$ expresses the unconditional transition from $c h a_{\text {init }}$ to $c h a_{\text {stop }}$ :

$$
\begin{aligned}
& \text { transf }_{1}:=\left(\text { cha }_{\text {init }} *(-,-) @ \text { time }, \text { cha }_{\text {stop }}\right) * \\
& \left((-,-),\left(\_,-\right)\right) @ \operatorname{strp} *\left(\left(\_,-\right),(,,-)\right) @ \text { @ick }
\end{aligned}
$$

That is, after the initialization phase (i.e. cha $a_{\text {init }}$ ) has taken place, we automatically end up in $c h a_{\text {stop }}$. Second, transf $f_{2}$ expresses that when pressing button STRP, a transition can take place from cha $a_{\text {stop }}$ to cha $a_{\text {start }}$ or vice versa:

transf $f_{2}:=t_{A} \wedge t_{B}$

with:

$$
\begin{aligned}
& t_{A}:=((\text { stop, },-),(-,-)) @ \text { mode } * \quad((0,1),(-,-)) @ s t r p \\
& \rightarrow \quad\left(\text { cha }_{\text {stop }} *(-,-) @ \text { @ick, cha } a_{\text {start }}\right) \\
& t_{B}:=\left(\left(\text { start }_{-}\right),\left(_{-},-\right)\right) @ \text { mode } * \quad((0,1),(-,-)) @ s t r p \\
& \rightarrow \quad\left(\text { cha } a_{\text {start }}, \text { cha } a_{\text {stop }} *(-,-) @ t i c k\right)
\end{aligned}
$$

Third, transf $f_{3}$ expresses that when button STRP is not pressed, the current mode stays the same: $\operatorname{transf}_{3}:=t_{C} \wedge t_{D}$

with:

$$
\begin{aligned}
& t_{C}:=\left(\left(\text { stop, },_{-}\right),\left(_{-},-\right)\right) @ \text { mode } * \sim\left((0,1),\left(_{-},-\right)\right) @ s t r p \\
& \rightarrow \quad\left(\text { cha }_{\text {stop }} *(-,,-) @ t i c k, c h a_{\text {stop }} *(-,,) @ t i c k\right) \\
& t_{D}:=\quad\left(\left(\text { start }_{-}\right),\left(_{-},-\right)\right) @ \text { mode } * \sim((0,1),(-,-)) @ s t r p \\
& \rightarrow \quad\left(\text { cha } a_{\text {start }}, \text { cha } a_{\text {start }}\right)
\end{aligned}
$$

Finally, the complete behavior of the Basic Stopwatch is formalized by: cha $a_{\text {basic }}^{2}:=$ transf $_{1} ;\left(\right.$ transf $_{2} \wedge$ transf $\left._{3}\right)$

\section{Basic Stopwatch with Reset}

We now enhance the behavior of the Basic Stopwatch by including the RESET button. Every time RESET is pressed, the Stopwatch re-initializes and starts executing from the beginning, i.e. from $c h a_{\text {init }}$. This modal behaviour is illustrated by the Cha ${ }^{4}$ Lo diagram in Figure 2 (iii) where the dotted box is a copy of Figure 2(ii), depicting the hierarchical mode $c h a_{b a s i c}^{2}$. The preemptive transitions, outside the box, have higher priority than the transitions inside the box. That is, pressing RESET has higher priority than pressing STRP. Every time RESET is pressed, the mode $c h a_{\text {init }}$ is re-executed. Formally:

$$
\begin{aligned}
\text { cha } a_{\text {reset }}^{4}:= & \left.\left.\left(\left(()_{-}\right),(0,1)\right),\left(()_{-}\right),\left({ }_{-},-\right)\right)\right) @ \text { reset } \rightarrow \\
& \left(\text { cha } a_{\text {basic }}^{2}, \text { cha } a_{\text {basic }}^{2}\right) \otimes\left(\left(\left({ }_{-},-\right),(-, \text {init })\right),\left(\left({ }_{-},-\right),\left(_{-},-\right)\right)\right) @ \text { mode }
\end{aligned}
$$

\section{Findings}

To conclude the Stopwatch case study, note that Separation Logic was not originally intended to express the modal behavior of a system, such as that of the Stopwatch. The above specifications seem to suggest, however, that Separation Logic may come in handy in at least two unexpected ways. First, the presented textual specifications denote the meaning of the graphical diagrams in Figure 2(ii) and (iii). These diagrams can be made (i.e. specified) by means of a graphical user interface. The corresponding graphical-specification process, in turn, could be a complementary (or competitive) alternative for the textual-based Esterel specification process. Second, since the presented logics elegantly capture modal behavior, they can of course also be used to provide an alternative formal semantics of languages such as Esterel [3]. 


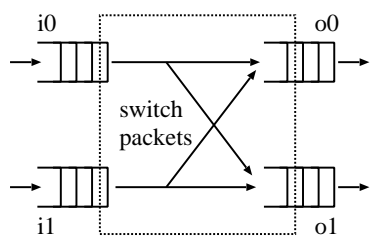

(i)

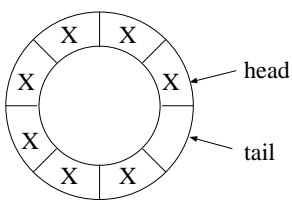

(ii)

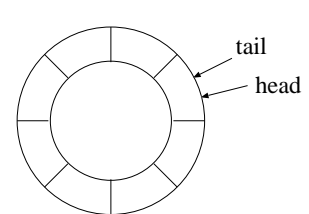

(iii)

Figure 3: (i) The $2 \times 2$ Switch, (ii) a full FIFO buffer, and (iii) an empty FIFO buffer.

\section{Switch}

Our third case study is a $2 \times 2$ Switch. Its shared-memory behavior was originally 3 specified in the guarded-command language Bluespec [2]. In this section, however, we introduce the Logic of Hierarchical Snapshots and reuse ChaLo (cf. Table 1) to specify the Switch's behavior.

This section consists of three parts. First, we present the design intent of the Switch in Section 4.1 Second, we introduce the Logic of Hierarchical Snapshots in Section 4.2 Finally, we partially specify the Switch's behavior in Section 4.3 .

\subsection{Design Intent}

The $2 \times 2$ Switch in Figure 3(i) contains two input FIFOs (i0 and i1) and two output FIFOs ( $\circ 0$ and $\circ 1$ ). A data packet can arrive on $i 0$ or i1. If the first bit of that packet has the value 0 , then it is routed to $\circ 0$, else to $\circ 1$. Each FIFO has the capacity to store 1021 data packets and 3 management packets (see below). Each packet contains 32 bits. A data packet can only move if the output FIFO is not full. A shared resource collision can occur when the data packets at the head of both input FIFOs have the same destination buffer (i.e. shared memory). In this case, i0 is given priority and i1's data packet is delayed.

The three management packets (of each FIFO) are the head and tail pointers and the empty entry in Figure 3 (ii). The head pointer refers to the entry in the FIFO that contains the head data packet (if any). The tail pointer refers to the first empty entry. To distinguish a full FIFO from an empty FIFO (cf. Figure 3 (iii)), one buffer entry is not used to store a data packet. This entry, hence, stores the third management packet of the FIFO. We also mention that the head and tail pointers are stored in buffer entries 1022 and 1023, respectively.

\subsection{Logic of Hierarchical Snapshots}

The Logic of Snapshots has the purpose to concisely describe hierarchical storage. We present examples below, omitting the obvious but lengthy formal definitions.

Suppose input buffer i0 is assigned to photographer $\mathrm{Ph} 1$. Then Ph1 can zoom in on, say, entry number 3 of $i 0$ and take a snapshot of the stored packet pack. If pack resembles the number five, then Ph1 observes 5@i0.3. Ph1 can zoom in further by taking a snapshot of, say, the first two bits of pack. Ph1 would then observe: $1 @ i 0.3 .0 * 0 @ i 0.3 .1$. The first conjunct expresses that the very first bit (index 0 ) has the value one. The second conjunct states that the second bit (index 1) has the value zero. This indeed corresponds to the bit notation of the number 5 , which is $0 \ldots 0101$ with the least significant bit

\footnotetext{
${ }^{3}$ If the reader is unfamiliar with Bluespec, he or she can also think of $\mathrm{TLA}^{+}[11]$ as an alternative specification language for the $2 \times 2$ Switch.
} 


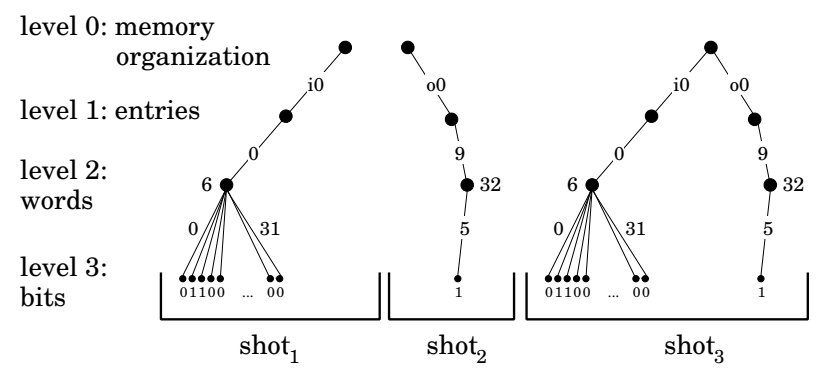

Figure 4: Three semantic snapshots.

(index 0) being the rightmost bit. If $\mathrm{Ph} 1$ chooses to observe $d=10$ consecutive bits of pack, starting from bit index $n=1$, then we may write 2@i0.3.1-10 because these ten bits, $0 \ldots 010$, resemble the number two. The general notation is: $v @ i 0.3 . n-n^{\prime}$ with $n^{\prime}:=n+d-1$, and where $v$ is the corresponding value. Finally, note that $\mathrm{Ph} 1$ can also combine disjoint snapshots of $i 0$ as for example:

$1 @ i 0.3 .0 * 0 @ i 0.3 .1 * 29 @ i 0.8 * 9 @ i 0.12 .4-17$.

\section{The Semantics of Hierarchical Snapshots}

Snshot, the domain of semantic snapshots for the $2 \times 2$ Switch, is defined in terms of Tree, a parameterized semantic algebra: shot $\in$ Snshot $:=$ Tree $[4,1024,32]$

The first parameter refers to the 4 buffers in Figure 3(i). Each buffer contains 1024 entries of 32 bits each.

Instead of giving a lengthy definition of Tree, we illustrate three semantic snapshots in Figure 4 For example, shot 2 is the semantic snapshot that models the syntactic snapshot $1 @ o 0.9 .5$.

Some more concepts follow. A path is a concatenation of edge numbers, such as o0.9.5. The trace of a tree shot is the set of paths that characterize all the level 3 nodes (i.e. bits) of shot. E.g.:

$\operatorname{Trace}\left(\right.$ shot $\left._{1}\right)=\{i 0.0 .0, i 0.0 .1, \ldots, i 0.0 .31\}$

Trace $\left(\right.$ shot $\left._{2}\right)=\{o 0.9 .5\}$

Two trees are disjoint $(\sharp)$ iff their traces are disjoint:

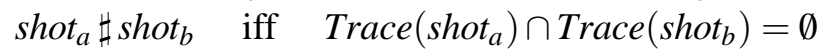

Thus, shot $t_{1} \sharp$ shot $_{2}$ holds. Since shot ${ }_{1}$ and shot $_{2}$ are disjoint, they can be combined (.) into shot $_{3}$ as follows:

shot $_{3}=$ shot $_{1} \cdot$ shot $_{2} \quad$ with

Trace $\left(\right.$ shot $\left._{3}\right)=$ Trace $\left(\right.$ shot $\left._{1}\right) \cup \operatorname{Trace}\left(\right.$ shot $\left._{2}\right)$

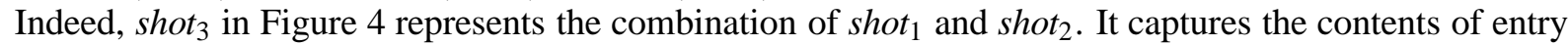
number 0 of buffer i 0 and bit number 5 of entry number 9 of buffer 00 . Finally, when two non disjoint trees are combined, then $\perp$ is returned. E.g.: shot $_{1} \cdot$ shot $_{1}=\perp$.

\subsection{Some Specifications}

In conformance to the hierarchical snapshots, presented in the previous section, we now present ChaLo specifications of the Switch.

As a first example, we want idleInputBuf $[$ buf $]$ to state that no packet is taken out of input buffer buf -where buf is $i 0$ or $i 1$. In accordance to Figure 3 (ii-iii), we therefore want to specify that buf's head pointer head does not change. Since head is stored in buf.first, we write the following where buf is $i 0$ 
or $i 1$ :

(i) idleInputBuf $[$ buf $] \equiv \exists$ head. (head,head) @ buf.first

As a second example, we want retrieveFromBuf $[$ buf $]\left[n_{1}\right]\left[n_{2}\right]$ [value], with buf equal to $i 0$ or $i 1$, to state that: value value corresponds to value@buf.head. $n_{1}-n_{2}$ where head is the head pointer of buf. That is, we want to retrieve (but not extract) the $n_{2}-n_{1}+1$ bits, starting from index $n_{1}$, from the head data packet in buf. Formally, we have:

(ii) retrieveFromBuf $[$ buf $]\left[n_{1}\right]\left[n_{2}\right][$ value $]$

(iii) $\equiv \exists$ head. ( (head,_) @ buf.first

(iv) * (value,_) @buf.head. $\left.n_{1}-n_{2}\right)$

Note that (iii) does not specify the new contents of buf.first and (iv) does not specify the new contents of buf.head. $n_{1}-n_{2}$.

Based on (ii), we can now define the following:

(v) retrieveFromBuf $[$ buf $][$ value $] \equiv$ retrieveFromBuf $[$ buf $][0][31][$ value $]$

where buf is $i 0$ or $i 1$. That is, value represents the complete data packet that is stored at the head of buf.

As a third example, we want extractFromBuf [buf] $\left[n_{1}\right]\left[n_{2}\right][$ value], with buf equal to $i 0$ or $i 1$, to be similar to retrieveFromBuf $[$ buf $]\left[n_{1}\right]\left[n_{2}\right][$ value], except that we now not only retrieve but also extract the data bits from the head packet in buf. Formally:

(vi) extractFromBuf $[$ buf $]\left[n_{1}\right]\left[n_{2}\right][$ value $]$

(vii) $\equiv \exists$ head. ( (head, $(1+$ head $) \bmod 1022) @ b u f . f i r s t$

(viii) $\quad *$ (value, _) @ buf.head. $\left.n_{1}-n_{2} \quad\right)$

Note that in (vii) we now do specify the new contents of buf.first.

Based on (vi), we can define the following where buf is $i 0$ or $i 1$ :

(ix) extractFromBuf [buf] [value] extractFromBuf [buf] [0][31] [value]

An additional remark is that, constraints, such as:

(x) $\exists x$.extractFromBuf $[$ buf $][x] \rightarrow$ notEmptyBuf $[$ buf $]$

also have to be specified. In words, (x) states that extracting a packet $x$ from buffer buf implies that buf is not empty. The trivial definition of notEmptyBuf is omitted.

As a fourth example, consider:

(xi) (depart : x,0)@i0 $\equiv$ extractFromBuf $[i 0][0][31][x]$

$\otimes$ extractFromBuf $[i 0][0][0][0]$

It states that i0's head packet $x$ is extracted from the buffer and that it's first bit has the value 0 . Similarly:

(xii) (arrive : $y, 0) @ o 0 \equiv$ insertInBuf $[o 0][0][31][y]$

$\otimes \quad$ insertInBuf $[o 0][0][0][0]$

The definition of insertInBuf is omitted from this paper.

Based on the above, we now define:

(xiii) $\exists z .(($ depart $: z, 0) @ i 0 \rightarrow($ arrive $: z, 0) @ o 0)$

This expresses, amongst other things, that the departed packet $x$ and the arrived packet $y$ are one and the same packet $z$. Finally, consider:

(xiv) $\exists z .(($ arrive $: z, 0) @ o 0 \rightarrow($ depart $: z, 0) @ i 0 \vee($ depart $: z, 0) @ i 1)$

The arrival of a packet at 00 implies its departure from i0 or i1. Continuing in this manner, we can completely capture the Switch's behavior.

\section{Findings}

To conclude the $2 \times 2$ Switch case study, note that Separation Logic is typically used to verify pointerintensive code [13, 15]. Since the Switch also contains pointers, it is less surprising, compared to the 
Stopwatch case study, that Separation Logic can be used as a specification language for shared-memory systems such as the Switch.

\section{Conclusions \& Future Work}

We have captured the concurrent behavior of three very different systems by means of Separation Logic and its natural extensions. Instead of specifying a modal-based system in Esterel and a shared-memory system in Bluespec, we are now able to specify both systems by means of the same formalism -not to mention the Subway system which was originally specified in LUSTRE. That is, we have a unifying framework for multiple design approaches that initially seemed disparate. Alternatively, we could (in future work) provide a semantics for LUSTRE, Esterel, and Bluespec in our unifying formalism.

Critics may remark that any other specification language, say Esterel, can also be used to capture the behavior of any of the three presented systems. Hence, they might question the relevance of the formalism, presented in this paper. We respond in the two following ways.

First, we have provided insight into how three seemingly independent concurrent systems are related to each other: (i) the Switch's behavior merely differs from the Subway's behavior in that it requires hierarchical snapshots instead of plain snapshots, and (ii) the Stopwatch's behavior merely differs from the Subway's in that it requires change of change (and change of change of change) to be specified instead of only change. Now, in our formalism, anything that can be expressed with plain snapshots can also be expressed with hierarchical snapshots. Similarly, anything that can be expressed with change (cf. ChaLo) can also be expressed with the more powerful concept of change of change (cf. Cha ${ }^{2} \mathrm{Lo}$ ), etc. So, all three concurrent systems, presented in this paper, can be expressed in one and the same formalism which we denote here (for the first time) by: $\mathrm{Cha}_{3}^{4} \mathrm{Lo}$, which is an instantiation of $\mathrm{Cha}_{h}^{\mathrm{n}} \mathrm{Lo}$. The parameters $\mathrm{n}$ and $\mathrm{h}$ denote the number of changes and the hierarchical depth, respectively. For example, $n=h=1$ for the Subway, $n=4$ and $h=1$ for the Basic Stopwatch with Reset, and $n=1$ and $h=3$ for the Switch.

The potential power of our formalism $\mathrm{Cha}_{\mathrm{h}}^{\mathrm{n}} \mathrm{Lo}$ lies in being able to select a specific subset, defined by the values of $\mathrm{n}$ and $\mathrm{h}$, for a given application domain.

Second, we invite the reader to check whether the other specification languages (e.g. Esterel) can in fact capture the behavior of all three concurrent systems in a uniform and sufficiently concise way. As mentioned in the introduction, practitioners will typically not use Esterel to specify a shared-memory system and will not use Bluespec to specify the modal behavior of e.g. a Stopwatch.

Finally, in line with this paper, we also refer to our complementary work [5] in which we have applied different specification languages (including Bluespec) to one and the same case study (i.e. the $2 \times 2$ Switch case study).

\section{Acknowledgement}

This work was partially supported by an AFOSR grant and NSF grant CCF-0702316.

\section{References}

[1] A. Ahmed, L. Jia, D. Walker, "Reasoning about Hierarchical Storage", LICS'03.

[2] "Automatic Generation of Control Logic with Bluespec SystemVerilog", Feb. 2005 Bluespec, Inc., Available at: bluespec.com/products/Papers.htm 
[3] G. Berry, G. Gonthier, "The Esterel Synchronous Programming Language: Design, Semantics, Implementation”, Science of Computer Programming, 1992.

[4] L. Caires, L. Cardelli, “A Spatial Logic for Concurrency”, TACS 2001, pp. 1-37.

[5] E.G. Daylight, S.K. Shukla, "On the Difficulties of Concurrent-System Design, Illustrated with a 2x2 Switch Case Study", accepted for Formal Methods 2009, Eindhoven, the Netherlands, November 2-6, 2009.

[6] W.J. Fokkink, "Introduction to Process Algebra", Texts in Theoretical Computer Science, Springer, 2000.

[7] N. Halbwachs, "Synchronous Programming of Reactive Systems", Kluwer'93.

[8] N. Halbwachs, F. Lagnier, C. Ratel, "Programming and verifying real-time systems by means of the synchronous data-flow language LUSTRE”, IEEE Transactions on Software Engineering'92.

[9] D. Harel, "Statecharts: A Visual Formalism for Complex Systems", Science of Computer Programming, 1987, 8, pp. 231-274.

[10] C.A.R. Hoare, “Comm. Seq. Processes”, Comm. ACM 21, 1978, pp. 666-677.

[11] L. Lamport, "Specifying Systems: The TLA ${ }^{+}$Language and Tools for Hardware and Software Engineers", Addison-Wesley Professional, 2002.

[12] R. Milner, "Communicating and Mobile Systems: the $\pi$-calculus", Cambridge University Press, 1999.

[13] P.W. O’Hearn, J.C. Reynolds, H. Yang, "Local Reasoning about Programs that Alter Data Structures", CSL'01.

[14] D. Potop-Butucaru, R. de Simone, J-P. Talpin, "The Synchronous Hypothesis and Synchronous Languages", in R. Zurawski, ed., "The Embedded Systems Handbook", CRC Press, 2005.

[15] J.C. Reynolds, “Separation Logic: A Logic for Shared Mutable Data Structures.”, LICS'02.

[16] H. Yang, "Relational Separation Logic", Theoretical Computer Science, Vol. 375, Issue 1-3, May 2007, pp. 308-334. 